\title{
La quadrature du texte ou l'énigme des quatre Le Quatrième Côté du triangle de Sony Labou Tansi
}

\author{
Nicolas Martin-Granel
}

\section{OpenEdition}

Journals

\section{Édition électronique}

URL : http://journals.openedition.org/genesis/603

DOI : 10.4000/genesis.603

ISSN : 2268-1590

Éditeur :

Presses universitaires de Paris Sorbonne (PUPS), Société internationale de génétique artistique littéraire et scientifique (SIGALES)

\section{Édition imprimée}

Date de publication : 30 octobre 2011

Pagination : 53-66

ISBN : 978-2-84050-804-5

ISSN : 1167-5101

\section{Référence électronique}

Nicolas Martin-Granel, « La quadrature du texte ou l'énigme des quatre Le Quatrième Côté du triangle de Sony Labou Tansi », Genesis [En ligne], 33 | 2011, mis en ligne le 23 octobre 2013, consulté le 19 avril 2019. URL : http://journals.openedition.org/genesis/603; DOI : 10.4000/genesis.603

Ce document a été généré automatiquement le 19 avril 2019.

Tous droits réservés 


\title{
La quadrature du texte ou l'énigme des quatre Le Quatrième Côté du triangle de Sony Labou Tansi
}

\author{
Nicolas Martin-Granel
}

J'essaye de créer, créer sans maître, créer sans
principes,
à la manière de Dieu - créer presque sans élan,
dans un délicieux désordre.
Sony LABOU TANSI

\section{Dans le labyrinthe}

1 L'œuvre de Sony Labou Tansi fuse dans tous les genres et dans tous les sens. Tel le jardin borgésien « aux sentiers qui bifurquent ", elle se présente à nous comme un labyrinthe. À nous lecteurs mais aussi, et d'abord, à l'auteur lui-même puisqu'il est de son travail en cours le premier lecteur, le premier critique :

Je ne sais pas dans l'édifice, ce qui craque, ce qui tient, ce qui part, ce qui reste, ce qui boude, ce qui ment, ce qui trahit... Je voudrais l'édifice. Qu'il gicle dans le ciel, chaque mot étant une pierre, un frontoglyphe, des labyrinthes incessants, voilà ce qu'est la création. Le temps aussi tient du labyrinthe. Comme la vie. Labyrinthes emmêlés, embrouillés ${ }^{1}$...

2 Or cet aveu d'incertitude, Sony le fait à propos de son dernier roman en cours d'élaboration, Le Commencement des douleurs, roman dont on sait qu'il l'a beaucoup retravaillé tout au long de ses multiples versions ${ }^{2}$, entre la première en 1989 et sa publication posthume en 1995. Il avoue ses doutes, ses hésitations, ses « affres » d'écriture non pas devant la page blanche, mais sur la difficulté à achever un texte, à en sortir, une fois franchi le seuil du titre qui en constitue «le premier pas $^{3}$ ». Et il se trouve qu'à son insu la métaphore du labyrinthe trouve sa vérité une fois l'œuvre achevée puisque ce n'est pas seulement le roman en gestation qu'il s'agit d'enfanter dans de telles douleurs 
mais bien l'édifice entier qui avec ce roman posthume reçoit sa dernière pierre, sa configuration définitive.

3 À s'en tenir à l'œuvre romanesque, qui forme tout de même un édifice assez considérable puisqu'il « gicle vers le ciel » à la hauteur de six romans, et si on jette sur ceux-ci un coup d'œil rétrospectif, on aperçoit très vite qu'ils ne sont pas coulés d'un seul bloc, encore moins extraits de la même veine. De fait, les six romans se laissent volontiers regrouper en deux séries symétriques d'égale importance, disons deux cycles de trois romans caractérisés par une certaine homogénéité du matériau lexico-sémantique et du système énonciatif adoptés. Le premier, qui comprend, dans l'ordre d'écriture, L'Anté-peuple, La Vie et demie et L'État honteux (alias Machin la Hernie), est écrit à la troisième personne et traite de façon plus ou moins réaliste ou grotesque d'un état social et d'un pouvoir politique particulièrement « moche » et mâle, tandis que le second (Les Sept Solitudes de Lorsa Lopez, Les Yeux du volcan, Le Commencement des douleurs) évoque un autre monde, à l'intérieur d'une géographie utopique qui redessine une nouvelle carte du tendre, nettoyée comme par magie du sexe de la honte ; ce monde à présent replacé sous l'empire du féminin et du divin, dominé par l'amour des femmes, s'écrit à la première personne du pluriel, le «nous » de la communauté originelle. Tels sont grosso modo, grossièrement tracés, les traits différentiels qui composent les deux massifs de l'édifice fictionnel. Dès lors se pose la question de savoir comment la configuration de ce double univers pourrait se penser au sein d'une création continue, quelles sont les modalités de passage de l'un à l'autre ; ou alors s'il y a bien mutation du paradigme, quel est le seuil à partir duquel Sony Labou Tansi se lance, après L'État honteux, dans une seconde révolution romanesque. Notre fil d'Ariane sera la femme, ou plutôt la question du féminin, puisque c'est elle, en définitive, qui permet d'échapper à la monstruosité mâle du Minotaure, autrement dit au ventre de l'État (hernie forcément sale, honteuse) en retournant dans le ventre (matrice forcément sacrée, honorable) de la nation. Révolution éthique et politique autant qu'esthétique. Arrivé au bout de L'État honteux, venant à bout de l'Enfer de la «viande » et de «la hernie", après en avoir exploré la "condition honteuse", inhumaine, cynique et diabolique, le moraliste Sony Labou Tansi paraît découvrir la complicité essentielle, naturelle et quasi étymologique du pouvoir (politique) sur les hommes et de la puissance (sexuelle) :

Le cœur d'un homme est dans la flotte qu'il te jette, comme le cœur du ciel est dans la pluie. La flotte tu comprends, tous les hommes c'est d'abord et avant tout une manière de flotter 4 .

Cette considération désabusée sur la domination masculine, l'écrivain la place fictivement dans une bouche de femme s'adressant à une autre femme. Ce n'est certes pas la première fois que Sony donne la parole à une femme, mais jusqu'ici elle n'était que personnage. Mais ici, il lui donne non seulement la parole mais aussi la voix : la femme est devenue narratrice et qui plus est de sa propre histoire. Ce cas de narration autodiégétique féminine est un véritable hapax dans l'œuvre de Sony Labou Tansi ; à cet égard il nous semble une pièce essentielle du puzzle sexuel et textuel, un fil d'Ariane pour se diriger dans le dédale, et notamment pour comprendre le passage du premier au second cycle romanesque. 


\section{Un titre en quête de texte}

5 Cet hapax remarquable se trouve sous le titre Le Quatrième Côté du triangle. Il s'agit d'un roman inédit et inachevé dont seul un chapitre a été publié5. Mais avant d'y revenir plus longuement, il faut nous arrêter sur ce titre qui lui aussi est « piège » et pas uniquement par sa polysémie et son hermétisme mais parce que sa fonction identificatoire est notablement perturbée par la pluralité des individus textuels auquel il réfère. Sony Labou Tansi a en effet multiplié les textes sous ce même intitulé. Essayons d'en débrouiller l'écheveau.

6 En plus de ce roman inachevé auquel je viens de faire allusion, il existe trois autres textes homonymes : un recueil de poèmes, une nouvelle et un brouillon de genre indéterminé. C'est donc à quatre reprises que Sony use du même titre. La coïncidence du chiffre cardinal des textes et du chiffre ordinal du titre est troublante. Qu'elle soit ou non fortuite importe peu, en tout cas moins que l'inflation qualitative d'une telle formule dans la pensée et l'écriture de son auteur. Il est indéniable qu'il est hanté par ce titre dont il se sert à quatre reprises. D'autant que ces « reprises » ne concernent pas diverses versions ou ébauches d'un même texte en gestation. Non, il s'agit bien de quatre textes différents, autonomes, presque hétéroclites, tant par leur teneur thématique que par leur affiliation générique. Il est difficile de dater ces textes avec précision, tout au plus essaierons-nous de les situer dans leur successivité, à partir d'indices internes et externes.

7 La genèse de l'œuvre sonyenne ne se fait pas par des réécritures telles que la critique génétique a l'habitude d'en traiter mais dans et par une progression de textes indépendants, qui n'ont explicitement en commun que leur titre. Ce titre mana a-t-il un pouvoir poétique matriciel ou n'est-il qu'un indice invitant à chercher certaines similitudes ou convergences troublantes? Nous reviendrons à la fin de ce parcours macrogénétique sur la réponse possible à donner à cette interrogation.

8 Avec ces quatre Quatrième Côté..., on se trouve vraiment au cœur du labyrinthe. Entrons un peu plus avant, en les présentant, l'un après l'autre, dans le beau désordre de leur apparition improbable.

\section{Du côté de la nouvelle}

9 Partons simplement pour commencer de l'hypothèse que la centralité dans ces textes de la question du féminin et le retour du même titre fédérateur poussent à penser que Sony Labou Tansi cherche obstinément la juste expression poétique d'une même interrogation lancinante.

Le premier Quatrième Côté... amorce la métamorphose du personnage féminin en femme personne, sujet à part entière de son cœur, de son corps et de son sexe. Il s'agit d'une nouvelle dactylographiée inédite. En à peine neuf pages, elle raconte la vengeance d'une femme humiliée, Daïma, par son amant, Victor, qui lui refuse la considération publique qu'elle estime due en échange du don privé de sa " croupe ». L'incipit expose d'emblée le point de vue de la femme en posant en termes crus les termes du contrat rompu:

Daïma prit un autre verre. Elle vida le verre à la santé de cet homme, ministre fraîchement nommé mais qui ne l'avait pas invitée ce soir-là à la soirée des

félicitations. [...] Elle pensa qu'on n'invite pas les ordures du quartier à une soirée où l'on fête un ministre tout neuf, « et pourtant je lui montre bien ma croupe ", elle 
pensa à ses yeux pleins d'appétit, à ses mains, à ses lèvres, à tout ce qui sur son corps portait le sceau de leurs sexes, elle dit son nom, très doucement: Victor ; la colère lui serrait la gorge, c'est des cochons les hommes, ils n'auront jamais plus d'autre cœur que leur eau de merde, elle but un autre verre, " comme d'habitude ", dit-elle au garçon en se levant - il payerait, la bouffe et la boisson, la gueule.

11 Mais cette première vengeance, limitée au plan économique, ne suffit pas à satisfaire la colère de la femme et à laver l'affront, car celui-ci est aussi bien social et politique. En rester là serait confirmer son statut ordinaire de femme entretenue. Or Daïma pense la guerre des sexes avec la lutte des classes ; elle n'évalue pas seulement les rapports sexuels comme la contrepartie réelle de rapports d'argent mais elle les analyse aussi dans l'ordre symbolique du pouvoir social et politique. C'est pourquoi elle trompera son amant avec son chauffeur - et Sony souligne le caractère social de cette vengeance en substituant mademoiselle à madame, terme en effet plus flatteur pour une femme libre (la ndumba, héroïne de la chanson congolaise) et qui la sort de l'ordinaire : " c'était la première fois que le chauffeur l'appelait "mademoiselle", elle dégusta l'appellation avec une certaine fierté ». Sur le plan de la rivalité politique, elle triomphera enfin publiquement devant le président en personne, qui lui a demandé de mettre à son service son ancien talent de chanteuse.

Car ce personnage féminin de Sony Labou Tansi possède, comme son auteur, le don du verbe «magique ». C'est ici que nous entrons de plain-pied dans les mystères du fameux triangle et de son quatrième côté. Son côté le moins mystérieux serait à chercher dans la bizarre confusion des sexes qu'il semble appeler. Remarquons d'abord, dans la phrase de l'incipit déjà citée, la curieuse équivoque provoquée par le pluriel - « ce qui sur son corps portait le sceau de leurs sexes", d'autant plus curieuse en effet qu'on ne peut la prendre pour un lapsus calami de l'auteur puisque c'est lui-même qui de sa main a substitué le pluriel au singulier - une des rares variantes de lecture significatives sur l'ensemble du texte, avec celle portant sur l'hésitation madame/mademoiselle. Du coup, le sens de celuici s'obscurcit en ce point précis où un seul corps se voit scellé par deux sexes. Il faudrait donc admettre que ce corps viril, revu et corrigé avec les yeux d'une femme, paraît déjà, sinon vraiment androgyne, au moins en voie d'hybridation et d'indifférenciation. Tout se passe comme si dans l'affrontement du masculin et du féminin, leur frontière se brouillait et les sexes devenaient interchangeables. Un autre symptôme de ce brouillage peut se lire dans un autre lapsus, mais un lapsus linguae celui-ci puisqu'on le rencontre dans le discours de Daïma :

[...] mais après tout je lui montre bien mes couilles, je lui montre toute ma croupe, il faut bien qu'il voit aussi mon cœur, comment c'est fait là-dedans, parce qu'après les couilles, j'ai quand même un dedans et un vrai...

La femme se représente avec des attributs typiquement virils, qu'elle confond sciemment avec ceux que lui attribue le désir des hommes, justement parce qu'elle veut confondre l'homme en lui montrant qu'elle n'est pas qu'un objet à exhiber. En fait elle banalise les sexes extérieurs pour introduire un troisième terme - le cœur - qui s'oppose à eux, les dépasse en ce qu'il serait, lui, spécifiquement féminin.

Dans ce Quatrième Côté du triangle qui fonctionne comme un avant-texte du roman, le corps premier est désormais celui de la femme, désigné par le métonyme "croupe »; à l'homme est imputée une double marque négative, aussi bien comme porteur de «cerveau » que de «couilles ». C'est à ce double titre que la honte retombe sur lui. La femme au contraire semble douée d'une troisième dimension qui sauve l'honneur de l'humanité : en plus de sa tête et de sa croupe, elle a un cœur, mieux un poème («je 
n'avais plus de cœur mais un poème à la place du cœur »), autrement dit un "dedans » inséparable du corps extérieur, mais qu'il lui faudra montrer et dire par une sorte d'invagination mystique où l'invisible deviendra visible, l'intérieur extérieur.

Plus tard, dans le roman qui deviendra Les Sept Solitudes ${ }^{6} . .$, la narratrice posera de façon encore plus explicite et provocante l'équivalence paradoxale entre le sexe le plus intime de la femme et le monument le plus sacré de l'homme : «Vraiment ! Le vagin, j'en fais un temple: c'est mon choix. Je peux tout souiller, sauf lui, mon vagin» (p.191). Et ce sanctuaire aux multiples côtés (sexuel et religieux bien sûr mais aussi politique et poétique), on le verra se dresser à nouveau dans Les Sept Solitudes..., mais évoqué cette fois dans toute la splendeur de son architecture composite et baroque :

Qui saura jamais ce qu'est le corps ? Le mien pourtant je le devine agréable à toutes les folies du monde, bâti à la seule mesure de la démesure. [...] Corps de turbulences, avec ses dômes, flèches, corniches, labyrinthes, mâchicoulis, minarets... C'est justement la chair qui en disant " je », change l'univers en un inépuisable chant de triomphe (p. 49).

16 À la fin de la nouvelle, soit au moment où, tel un deus ex machina, le président par un coup de téléphone providentiel va permettre à Daïma d'accomplir sa vengeance, s'accomplit aussi une sorte de coup d'état énonciatif :

Comme la nature sait régler ses comptes: le président veut que je chante pour l'anniversaire du régime samedi. Ils ont tout fait pour retrouver mon identité. C'est lui, Victor qui a voulu m'avoir toute à lui, il m'a donné une autre vie que celle de danseuse qui chante pour allumer le désir de tous. " Tu ne seras plus Gama Rabaldi, mais l'ange de mes amours. Tu t'appelleras Daïma, mon cœur, ma force et ma vie. Tu seras le quatrième côté du triangle que je forme avec ma femme et mon fils.» [...]

Pendant trois jours j'ai retravaillé sur mon répertoire que j'ai intitulé « cours de dignité »: sept chansons au total: la première se nomme l'âge d'or [...] et la dernière le quatrième côté du triangle.

17 Ainsi à l'heure des règlements de compte pour son personnage, Sony règle aussi les siens. Avec la voix narrative d'abord: alors que celle-ci normalement reste stable d'un bout à l'autre du récit, il saute ici brusquement, d'un paragraphe à l'autre, de la troisième à la première personne; « je » a pris le contrôle de la narration et ne le lâchera plus : dans le dernier tiers de la nouvelle, la non-personne « elle » n'apparaîtra plus du tout. Cette inconséquence est par elle-même, en tant qu'événement narratif, suffisamment signifiante, surtout quand on la met en regard de ce qu'il advient sur le plan proprement diégétique. Le personnage féminin se métamorphose. Pour être "je», étant "je», elle abandonne le nom de Daïma dont Victor, son amant victorieux, l'avait baptisée pour mieux la posséder, ainsi que l'attestent tous les possessifs dont son discours amoureux est saturé (mes amours... mon cœur, ma force, ma vie... ma femme et mon fils...) ; et ce rejet signifie qu'elle renonce au pseudo-titre de " quatrième côté... ", lequel recouvre en fait un simple statut de « deuxième bureau ». Dès lors que s'ouvre l'accès à la première personne, elle ne peut que s'arroger le premier rôle, le rôle-titre. Inversant les signes cryptés, renversant enfin le symbolisme du "quatrième côté ", elle fait de celui-ci le titre de sa septième et dernière chanson, de son premier grand rire, et finalement de son triomphe.

Comme son personnage féminin, l'auteur possède à l'intérieur les mêmes moyens : un poème à la place du cœur, une passion pour les changements d'identité, un goût de l'énigme, du chiffre. Soit le titre qu'il pose comme une énigme à déchiffrer. Victor croit en venir à bout par la doxa masculine d'une puissance virile doublée : «le quatrième côté » s'ajouterait en trompe-l'œil au triangle familial classique. Las, cette clé est un passe- 
partout qui ne saurait tromper la femme. Le quatrième côté est bien celui d'un carré invisible mais qui s'additionne aux trois côtés du triangle. Basculant entre deux figures non superposables, le chiffre mystique est amplifié jusqu'au nombre sept. On ne peut que le mettre en musique et en mots, en chanson et en poème. D'où les sept chansons au répertoire de Daïma alias Gama Rabaldi, un chiffre qui annonce Les Sept Solitudes..., nouvel alias du Quatrième Côté...

\section{Du côté du roman}

\section{unté, les corps amor} de ce tournant: la boue. Elle a changé de dénotation, tout en gardant la même connotation. Dans tout le cycle antérieur jusques et y compris la nouvelle, elle désigne la misère qui détermine les rapports, le commerce, entre les sexes. Ainsi, tandis qu'elle représente pour Daïma l'existence d'où son puissant amant l'a tirée et où il semble la renvoyer en ne l'invitant pas à partager sa réussite, la « boue » devient, dans le roman, la métaphore du corps à corps impur et obscur : «l'homme et la femme dans la boue du sexe ». Pour s'en extraire, il faudrait "travailler à l'avènement vers une nouvelle

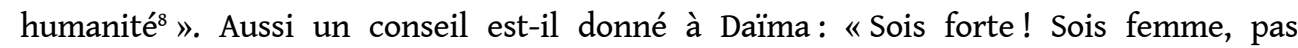
femelle» (p.64). Injonction dont la teneur est bientôt reprise en exergue des Sept Solitudes... : «Et que maintenant la femme se batte avec d'autres armes que la femme femelle ».

Le clivage femelle/femme est d'abord affaire de langage. Ce que montrent les corrections auctoriales qui jouent toutes précisément dans le même sens : gommer l'effet femelle et ajouter, corrélativement, une autre dimension à la figure triangulaire du sexe féminin. 
Dans le processus d'écriture à "structuration rédactionnelle " " de Sony Labou Tansi, l'opération de réécriture ne se fait quasiment jamais sur le même support matériel. S'il devait en effet retravailler sur un texte en chantier, Sony prenait un cahier vierge. Rares donc sont les cas où Sony revient sur du déjà écrit. Ces cas de réécriture sur le même cahier n'en sont que plus notables. Or il est remarquable que la plupart partent de la rhétorique sexuelle et aboutissent à donner des sexes et de leurs rapports une image moins crue. De ce phénomène d'euphémisation généralisée, voici quelques exemples significatifs, soit exactement sept variantes ${ }^{10}$ par rapport à la version publiée: suppression de « non pas un viol! » après « je n'ai pas rêvé... » (p. 63) et substitution de :

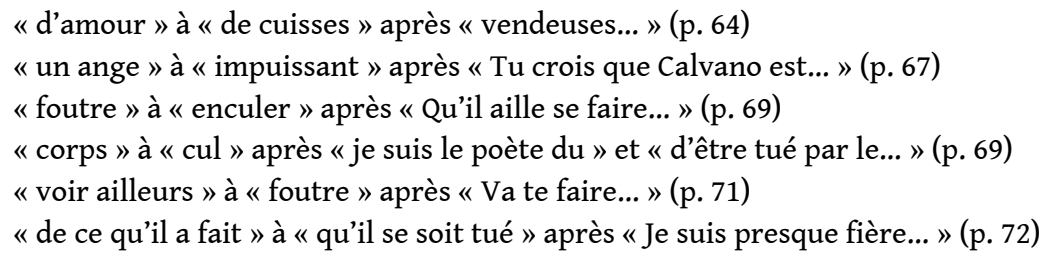

Ajoutées les unes aux autres et mises en perspective, toutes ces corrections aboutissent à corriger l'image de la femme. Par rapport à la Daïma de la nouvelle, encore "femelle ", Gracia, le dernier avatar romanesque, est un produit plus conforme au genre «femme ». En fait elle est la matrice d'où sortira, régénérée, la femme nouvelle, coulée dans le bronze d'Estina Bronzario. Dès lors il apparaît que la césure entre le cycle romanesque masculin et le nouveau cycle au féminin pluriel passe entre la nouvelle et le roman homonyme, entre la figure de Daïma et celle de Gracia.

Mais on sait que Sony ne donnera pas suite au roman Le Quatrième Côté..., sinon dans un autre genre ou encore sous un autre titre : Les Sept Solitudes de Lorsa Lopez.

\section{Du côté du poème}

27 C'est sans doute, paradoxalement, le plus visible, depuis la parution de Il quarto lato del triangolo, recueil de poèmes dont Sony remit le cahier manuscrit à son ami Sergio Zoppi en 1988, et que celui-ci publia, quelque dix ans plus tard, en édition bilingue et relativement génétique ${ }^{11}$. Parmi les quatre pages du cahier manuscrit reproduites en facsimilé, celle du frontispice porte la date du 25 septembre 1988.

Cependant il n'est sans doute pas sans intérêt de remarquer que dans l'Avertissement des Sept Solitudes..., dans le même temps où il affirme hautement l'altérité foncière de sa nouvelle démarche artistique, l'auteur se définit comme poète :

Dans ce livre, j'exige un autre centre du monde, d'autres excuses de nommer, d'autres manières de respirer... parce que être poète, de nos jours, c'est vouloir de toutes ses forces, de toute son âme et de toute sa chair, face aux fusils, face à l'argent qui lui aussi devient un fusil, et surtout face à la vérité reçue sur laquelle nous, poètes, avons une autorisation de pisser, qu'aucun visage de la réalité humaine ne soit poussé sous le silence de l'Histoire.

Décidément "ce livre", en s'annonçant comme autre, annonce l'exigence d'une autre poétique, une poétique qui redonne sa juste place, justement, à la poésie : au centre. Ce qui suppose de décentrer le roman de la "vérité reçue " vers la dimension inaperçue, passée "sous le silence de l'Histoire », du sujet lyrique, de la subjectivité. Nul doute que celui-ci ne soit de nature féminine. De la même façon qu'il est dit « La femme est le lieu exact de la naissance » (citation du poète Édouard Maunick mise en épigraphe du roman), on peut dire que la poésie doit être le lieu de la renaissance du roman sonyen. Pourtant, 
tout en écrivant en parallèle poèmes et romans, le "poète " propose, prophétise une géométrie non euclidienne qui, parce que l'ancienne est trop compromise avec la « réalité moribonde » d'une Histoire dépassée, ouvre sur le mélange des genres, en ce qu'il appelle « un silence métissé ». D'où le surgissement, à l'intérieur même du roman, d'un fragment de poétique non euclidienne :

Toutes nos histoires et tous nos racontars tentent de nous sortir de la géométrie tracée par cette réalité moribonde où nous enferment le dénuement matériel et la dévirginisation de notre conscience. La misère spirituelle est la plus bête de toutes les misères. C'est pour lutter contre elle que nous nous évertuons à inventer l'inflation des langages. Nous avons toujours réussi des échappatoires fulgurantes au tribunal des mots. Nous sommes le seul peuple au monde à avoir obtenu un nonlieu devant la dictature du verbe (p. 143).

«Géométrie... inflation des langages... échappatoires... non-lieu » : ne seraient-ce pas là les mots-clés du commentaire le plus autorisé pour comprendre la formule "Le quatrième côté du triangle »?

\section{Faux départ ou fausse sortie?}

31 Le dernier texte à porter ce titre est de nature très hybride, d'un genre improbable. Matériellement, il fait partie, semble-t-il, d'un début de roman intitulé Fabien Israël, et formé de onze feuillets d'écolier (grand format), manuscrits recto verso, dont il occupe les trois dernières pages. Les dix premières constituaient à l'origine le premier chapitre intitulé "Les criquets»; ce titre est biffé et le texte paraîtra sous le titre prévu initialement pour le roman, mais en tant que nouvelle ${ }^{12}$. Quant au " deuxième chapitre ", qui semble n'avoir aucun rapport thématique ou narratif avec ce qui le précède, il sera raturé de deux traits en diagonale. Voici la transcription du début du deuxième chapitre :

2 Le Quatrième côté du triangle

À mon ami Demba Ntelo

«Élever des murs c'est rigoler du temps qui finit toujours par les écrouler. Mettre un enfant au monde c'est se rire du temps qui finit toujours par le tuer. Élever des espoirs... Organiser... Protester... Militer... Croire... Tout ce que nous pouvons penser ou faire, le temps est méchant maître... rien ne se fait sans lui... » Mon frère et ami Jean-Claude est venu me voir pour me montrer son chantier. Nous avons traversé le Djoué par l'ancien pont. Le nouveau est en réfection et la population rigole de l'État qui ne fait jamais rien de solide. Il fait une chaleur d'argent. Le fleuve montre le dos noir de ses monstres de pierre sur lesquels les siècles ont toujours glissé. Fleuve de Kongo, disaient nos anciens. À cause de ces pierres sans doute. Elles semblent boire à toutes les coupes de la paix. Kongo c'est la paix. La paix du cœur pour certains ${ }^{13}$. La paix tout court pour le commun des mortels qui ne se mêle jamais ni d'étymologie, ni d'aucune autre espèce d'affiliation. Nous nous sommes arrêtés à Mafouta pour dire bonjour (le mot juste serait bon après-midi) aux gens qui construisent la tombe de la mère de notre ami Sylvain, morte mardi dernier. Sans doute parce qu'on me prend pour historiographe de la révolution on me montre tout, on me raconte tout. [...]

Page arrachée d'un journal intime? tentative de lever le voile sur la genèse de son écriture? essai autobiographique? poussée de roman-trottoir? mise à nu d'une philosophie nihiliste? aveu d'échec du roman? jeux étymologiques? Quoi qu'il en soit du genre, Sony Labou Tansi expérimente "d'autres manières de nommer ». Il met en abyme, dans la douleur et la dérision, le « chantier » de son œuvre labyrinthique. On y reconnaît sans peine des personnes - l'homme d'affaires Jean-Claude (Silou) ou l'écrivain Sylvain 
(Bemba) - et des toponymes attestés (Mafouta, le Djoué, les Rapides). Au-delà de cet ancrage autobiographique, ce texte bref met en crise le roman fondé sur le temps. Puisque celui-ci se révèle mortifère et "méchant ", le romancier va se choisir un nouveau «maître »: l'espace personnifié ici par le fleuve Congo et «ses monstres de pierre sur lesquels les siècles ont toujours glissé ». Plus solide que les éphémères constructions humaines, l'espace naturel fournit un socle dur, autochtone, sur lequel fonder la paix civile et politique et finalement recentrer le roman ("élever des murs») autour de ces nouvelles figures de vie. Et ce n'est pas un hasard si ce sont toutes des victimes du temps, si bien qu'au début Sony les confond et les subsume sous le nom du Juif (Fabien Israël).

Ce texte inédit est aussi un hapax, de par le caractère étrange et plurivoque de son écriture : intime mais en même temps vouée à la publication, ainsi qu'en témoigne son paratexte et en particulier la dédicace. Il invente un nouvel espace d'écriture investi, traversé par le prophétisme scripturaire de Sony qui se met à écrire des fictions comme autant de lieux de conjonction et d'inscription du livre (le Livre), du sexe (la Femme) et de la politique (le Kongo). C'est par tous ces "côtés ", nous semble-t-il, que son auteur l'a baptisé, lui aussi, Le Quatrième Côté du triangle. On y voit comment l'homme s'inscrit dans sa création, à la façon d'une quatrième dimension invisible mais sous-jacente. Le court espace de ce texte, l'auteur apparaît en tant qu'homme concret, historiographe. Or, s'il disparaît si vite, si la voie autobiographique tourne court, c'est que sans doute l'homme n'y est pas à sa place. Il ne mérite pas « de figurer dans un livre ». La vérité de l'homme peut être belle, elle ne suffit pas (plus ?) pour faire un livre. Il y faut une " autre vérité », non pas celle de l'amitié réelle mais celle de l'amour rêvé. Elle seule permet «d'inventer l'inflation des langages ». Pour la rendre, il y faudra donc un autre sexe, c'est-à-dire, à la lettre, devenir ${ }^{14}$ femme.

\section{Sorties}

Revenons sur les questions soulevées au début.

Après L'État honteux, Sony inaugure un nouveau cycle romanesque, recyclant l'obscène dans le secret, l'histoire dans la géographie et le réalisme dans l'utopie. Cependant ce recommencement, déjà douloureux, ne se fait ni sans mal ni sans ratés. Il a ainsi écrit, ou commencé d'écrire, quatre œuvres portant le même titre «Le quatrième côté du triangle ». Dont aucune ne verra le jour, de son vivant du moins.

De ces avortements successifs, on peut tout de même essayer, à l'aide des analyses précédentes, de retracer l'hypothétique chronologie. La nouvelle tout d'abord (1980 ?), puisqu'elle amorce le retournement de la femelle en femme, ainsi que sa saisie de la position narrative. Puis le roman qui constitue l'avant-texte des Sept Solitudes de Lorsa Lopez. En troisième lieu, je placerais le recueil de poèmes remis à Sergio Zoppi en 1988, et en dernière position l'essai de journal, qu'il est assez facile de dater grâce à l'indication de la mort de la mère de Sylvain Bemba: 1988. Peu après cet essai avorté d'écriture autobiographique, Sony Labou Tansi se lance dans Le Commencement des douleurs - un chantier interminable dont le premier brouillon, dès les premières lignes (dans l'Avertissement), est solennellement daté : « 8 mars 1989 ». Ce qui est une première dans l'écriture de Sony.

37 La curieuse homonymie de ces quatre avatars hétérogènes du Quatrième Côté... est révélatrice de la crise d'identité textuelle aussi bien que sexuelle que traverse l'auteur 
tout au long des années quatre-vingt. S'il est vrai qu'il n'a cessé de chercher à répondre à la question « qui suis-je ? » ${ }^{15}$, le premier cycle de romans se construit sur l'annihilation de la première personne : «N'allons pas fouiller pour qui est mis moi - on perdrait du temps. Moi $=$ personne. Mais pas rien, tu comprends ${ }^{16} . »$ Longtemps contenue dans le ventre du pouvoir politique et de la puissance masculine, la première personne revient cependant ensuite, mais sous un masque féminin. Ce nouveau sujet d'écriture se disséminera désormais dans tous les genres littéraires. Au théâtre, ce sera Moi, veuve de l'empire; en poésie, les « 100 femmes » du recueil 930 Mots dans un aquarium.

Reste le titre sibyllin dont j'ai usé comme d'un fil d'Ariane. C'est le chiffre du labyrinthe que Sony construit et où il « erre entre ce qu'on veut taire et ce qu'on veut dire ${ }^{17}$ ». Hanté et même fasciné par cette figure géométrique, figure de l'impossible et de l'innommable, il l'inscrit à quatre reprises comme une énigme au fronton du nouveau temple qu'il commence à édifier dans la douleur. Une seule fois, la première, il semble donner la clé de l'énigme : c'est dans la nouvelle où l'homme offre à sa maîtresse le statut de "quatrième côté »; elle en fera le chant de son triomphe. Mais cette géométrie amoureuse va bien audelà de « l'anecdote », dont Sony disait que ses titres étaient porteurs. Le titre renvoie à la poétique de l'excès (voir les titres La Vie et demie, Le Huitième Jour de la semaine $e^{18}$ ), comme pour dire qu'ici la femme excède son sexe. À l'arme traditionnelle de la femelle répond le culte du vagin en tant que «lieu exact de la naissance » et par conséquent creuset de la nation. Or la mise au monde de la nation, ou plutôt sa re-naissance, ne peut s'opérer que dans la souffrance d'un double symbole, politique et religieux : d'un côté le triangle du Kongo Ya N'tatu ( = trois) ${ }^{19}$ et, de l'autre, le carré qui est l'insigne porté par ceux qui se réclament du matsouanisme ${ }^{20}$. Conformément à l'interdit maternel proféré dans le premier essai romanesque, les deux parties du symbole ne sauraient donc jamais se superposer ni coïncider exactement. Sinon par ce "quatrième côté » dont on voit bien qu'il relève d'une autre géométrie, chargée de trouver une solution au différend du politique et du religieux, comme celui, tout aussi insurmontable, du masculin et du féminin. Au-delà de l'anecdote ésotérique, l'énigme titulaire n'a donc pas à se résoudre. Elle serait de l'ordre de l'ineffable, de l'inarticulé, bref du cri, comme le dit Sony dans un entretien $^{21}$ où apparaît d'ailleurs l'unique mention, à ma connaissance, du titre aux multiples tiroirs secrets :

Tous mes titres sont des cris, vraiment des cris, qui explosent dans La Vie et demie,

L'État honteux. [...] L'Anté-peuple, et tous ceux que tu veux, ce sont des cris, Le Quatrième Côté du triangle, Le Commencement des douleurs, qui est mon prochain livre sur lequel je suis en train de travailler maintenant.

Faisant ainsi se succéder les deux derniers titres, Sony ne laisse-t-il pas entendre que Le Commencement... serait la suite du Quatrième Côté... ? que celui-ci explique celui-là ? Quel que soit le sens dans lequel il faudrait déchiffrer ce palimpseste, il apparaît que les derniers textes entretiennent entre eux de forts liens d'hypertextualité. Et que l'énigme du Quatrième Côté du triangle est peut-être celle de toute écriture cubiste? 


\section{NOTES}

1. "Lettre à un étudiant mauritanien ", dans L'Autre Monde, Paris, Revue Noire, coll. "Soleil », 1997, p. 49.

2. Voir notamment Jean-Claude Blachère, Négritures, Paris, L'Harmattan, 1993, p. 226 sq., et JeanMichel Devésa, Sony Labou Tansi, écrivain de la honte et des rives magiques du Kongo, Paris, L'Harmattan, 1996, p. 367 sq.

3. Titre du premier roman envoyé au Seuil, voir mon «Le souffle et le travail », Études littéraires africaines, $\mathrm{n}^{\circ} 15,2003, \mathrm{p} .23 \mathrm{sq}$.

4. Sony Labou Tansi, L'Autre Monde, op. cit., p. 64.

5. Ibid., p. 62-72 sq.

6. Les Sept Solitudes de Lorsa Lopez, Paris, Éditions du Seuil, 1985.

7. Le manuscrit occupe soixante-dix pages sur deux cents dans un cahier d'écolier : deux pages de paratexte (reproduites en fac-similé dans L'Autre Monde, op.cit., p. 61), une pour l'avertissement, trente et une pour le premier chapitre, dix-sept pour le deuxième, dix-neuf pour le troisième, sans qu'on puisse décider si ce dernier était achevé ou non. Comme presque tous les manuscrits de Sony, celui-ci n'est pas daté. Cependant il est possible d'inférer l'époque approximative où le cahier a été utilisé, grâce à trois documents qu'il contient. Deux feuilles volantes glissées dans les pages blanches : une courte lettre signée d'Arlette Chemain et datée du 13 juillet 1982, et un petit article critique sur « Jazz et vin de palme » d'E. Dongala paru dans l'hebdomadaire Jeune Afrique du 4 août 1982. Enfin, écrite au crayon de papier de la main de Sony sur la dernière page du cahier, la distribution des rôles pour Simba Vita, une pièce de théâtre que la troupe de Sony, le Rocado Zulu Théâtre, a monté cette même année. À partir de ces dates concordantes, on peut donc dater l'écriture du roman de l'année 1982, soit à peu près un an après que Béatrice du Kongo eut été créée au Centre culturel français, le 29 octobre 1981. C'est dire l'importance que prennent à cette époque les grandes figures de femmes dans la fiction de Sony, sur le mode tant narratif que dramatique.

8. Lettre à Sergio Zoppi (1988), en fac-similé dans Il quarto lato del triangolo.

9. Voir Pierre-Marc de Biasi, La Génétique textuelle, Paris, Armand Colin, p. 33.

10. Réalisées après coup, comme l'atteste l'emploi d'un autre stylo et/ou d'une autre encre que l'originale. On peut supposer que Sony relisait son texte, et éventuellement le retouchait, avant de le continuer à partir du point où il s'était arrêté. La description matérielle prouve qu'il s'agit non pas de simples variantes d'écriture, mais bien de véritables variantes de lecture.

11. Sony Labou Tansi, Il quarto lato del triangolo (Le quatrième côté du triangle), Cura di Sergio Zoppi e Antonella Emina, Traduzione di A. Emina, Torino, La Rosa, coll. « Tracce », 1997.

12. Sony Labou Tansi, «Fabien Israël », Revue des Deux Mondes, novembre 1991, p. 208 sq.

13. Allusion à un fameux discours de Denis Sassou Nguesso, dans lequel il avait promis, en 1983, «la paix du cœur et la tranquillité des esprits».

14. Ce verbe deleuzien était d'ailleurs pour le jeune Sony une sorte de mot mana : « Il y a un seul mot qui me met en position de force, c'est DEVENIR. J'ai plus que tous les autres le temps, la chance et la force de devenir " (lettre à Françoise Ligier, 2 octobre 1974, dans L'Atelier de Sony Labou Tansi, éd. Nicolas Martin-Granel et Greta Rodriguez-Antoniotti, Paris, Revue Noire, vol. I, «Correspondance », p. 156).

15. Diogène à Brazzaville, film de Léandre-Alain Baker, 1998.

16. Lettre à F. Ligier, 18 février 1976, op. cit., p. 183. 
17. Dans cet exergue à une version manuscrite du Commencement..., Sony a substitué erre à tourne, jouant sur la polysémie du verbe dont est issue aussi bien l'errance que l'erreur.

18. C'est le titre d'une pièce de théâtre commencée et abandonnée en 1980.

19. À noter que les communiqués militaires de la rébellion dans la région du Pool faisaient figurer un triangle au-dessous de « République du Kongo ».

20. Mouvement messianique congolais qui tire son nom d'André Matsoua (1899-1942), prophète rebelle qui poussa les populations lari à refuser l'indigénat et l'impôt et fut jeté en prison (où il mourut) par les autorités coloniales.

21. Dans les extraits transcrits dans Études littéraires africaines, $n^{\circ} 15,2003$.

\section{RÉSUMÉS}

Dans l'œuvre labyrinthique, polymorphe et transgénérique, de Sony Labou Tansi, caractérisée par la génétique textuelle comme "à déclenchement rédactionnel » et par l'anthropologie comme relevant du "prophétisme scripturaire ", le paratexte occupe une place singulière, labile, quasi indépendante du texte qu'il est censé annoncer et auquel il semble faire concurrence. Cet article examine le cas emblématique d'un titre, "Le quatrième côté du triangle ", qui apparaît doublement énigmatique : titre à la fois Sphinx par sa teneur cryptée et Phénix par son emploi itératif au cours d'une période de plus d'une dizaine d'années. On le retrouve en effet dans la nébuleuse des cahiers manuscrits, non datés et largement inédits, de l'écrivain congolais, nommant au moins quatre textes différents, de taille, période et genre très hétérogènes : une nouvelle, un roman inachevé, un recueil de poèmes, une ébauche d'essai autobiographique. Le dossier génétique de cet hypertitre nous oriente sur la piste heuristique d'une écriture hypertextuelle, vers ce point aveugle où s'élabore, à coups de faux départs, le processus ré (tro)volutionnaire du passage continu de l'obscène masculin au secret féminin qui mène au titre final : Le Commencement des douleurs.

Sony Labou Tansi's work, labyrinthine, polymorphous and transgeneric, is defined by genetic criticism as "trigger writing" and by anthropology as belonging to "scriptory prophesying". In this work the paratext holds a singular and fragile place has a singular, fragile role, almost independent of the text it is supposed to introduce and with which it seems to vie. This article examines the emblematic case of a title, "The Fourth Side of a Triangle", which appears doubly enigmatic. This title is both a Sphinx by its cryptic content and a Phoenix by its repetitive use over a period of more than a dozen years. Indeed, it appears again in this Congolese writer's undated and largely unpublished manuscript notebooks. It is the title of at least four different texts, of varying length, period and genre : a short story, an unfinished novel, a poetry book, the draft of an autobiographic essay. The genetic dossier of this hypertitle leads us on the heuristic track of a hypertextual writing, towards this blind spot where with false starts the re (tro)volutionary process of this constant passing from the obscene masculine to the secret feminine leads to the final title: The Beginning of Pains.

En la obra laberíntica, polimorfa y transgenérica de Sony Labou Tansi, cuya escritura es caracterizada por la crítica genética como "pulsional" y ubicada por la antropología en la esfera del "profetismo escriturario", el paratexto ocupa un sitio particular, lábil, casi independiente del texto que en principio debe anunciar y con el cual parece entrar en competencia. Este artículo 
analiza el caso emblemático de un título, "El cuarto lado del triángulo", que resulta doblemente enigmático: título a la vez Esfinge, por su contenido críptico, y Fénix, por su utilización reiterativa a lo largo de un periodo de más de una década : aparece, en efecto, en la nebulosa de los cuadernos manuscritos, sin fechar y mayoritariamente inéditos, del escritor congolés, y más concretamente, en al menos cuatro textos diferentes, cuyas extensiones, periodos y géneros son tres heterogéneos: un cuento, una novela inconclusa, un conjunto de poemas, un esbozo de ensayo autobiográfico. El dossier genético de este hipertítulo nos conduce en la pista heurística de una escritura hipertextual, hacia ese punto ciego donde se elabora, a base de arranques fallidos, el proceso re(tro)volucionario del paso permanente de lo obsceno masculino a lo secreto femenino que conduce al título final : El comienzo de los dolores.

Im labyrinthischen, polymorphen, und genreübergreifenden Werk von Sony Labou Tansi, von der Textgenetik als „redaktionell ausgelöst“ und von der Anthropologie als „Prophetie der Schrift" charakterisiert, kommt dem Paratext eine einzigartige, schillernde, quasi vom Text unabhängige Stellung zu, auf welchen er eigentlich verweisen sollte und dem er Konkurrenz zu machen scheint. Dieser Beitrag untersucht den emblematischen Fall des Titels, „Le quatrième côté du triangle“, der doppelt rätselhaft erscheint : Sphinx zum einen wegen seiner kryptischen Formulierung und Phönix durch seinen wiederholten Einsatz während einer Periode von mehr als zehn Jahren. Man findet diesen nämlich wieder im Nebel der undatierten und zu großen Teilen unveröffentlichten Manuskripthefte des kongolesischen Schriftstellers, wo dieser wenigstens vier verschiedene Texte bezeichnet, die in Umfang, Zeitraum, und Genre sehr verschieden sind: eine Novelle, ein unvollendeter Roman, eine Gedichtsammlung, sowie die Rohfassung eines autobiographischen Essays. Das genetische Dossier dieses Übertitels bringt uns auf die heuristische Spur eines übertextlichen Schreibens, bis an den blinden Fleck, wo sich, nach einigen falschen Fährten, der re(tro)volutionäre Prozess der kontinuierlichen Passage maskuliner Obszönität zum Geheimnis des Weiblichen entbirgt, der uns zum endgültigen Titel leitet : Le Commencement des douleurs.

Nell'opera di Sony Labou Tansi - labirintica, polimorfa, sfidante la classificazione di genere definita dalla genetica testuale come "rilascio redazionale" e dall'antropologia come "profetismo scritturale", il paratesto occupa un posto singolare, labile, quasi indipendente dal testo che dovrebbe annunciare, tanto che sembra quasi fargli concorrenza. Qui si esamina il caso emblematico di un titolo, "Il quarto lato del triangolo", che appare doppiamente enigmatico : allo stesso tempo Sfinge per il suo tenore criptico e Fenice per il suo impiego iterativo nel corso di oltre una decina d'anni. Lo si ritrova in effetti nella nebulosa dei quaderni manoscritti, non datati e per la maggior parte inediti, dello scrittore congolese, a indicare quattro testi diversi, molto eterogenei per dimensione, periodo e genere : un racconto, un romanzo incompiuto, una raccolta di poesie, un abbozzo di saggio autobiografico. Il dossier genetico di questo "ipertitolo" ci orienta sulla pista euristica di una scrittura ipertestuale, verso quel punto cieco dove si elabora, a colpi di false partenze, il processo retro-rivoluzionario del passaggio continuo dall'osceno mascolino al segreto femminino che porta al titolo finale : Le Commencement des douleurs.

$\mathrm{Na}$ obra labiríntica, polimórfica e transgenérica de Sony Labou Tansi, caracterizada pela genética textual como de "desencadeamento redaccional" e pela antropologia como derivando de "profetismo scripturário", o paratexto ocupa um lugar singular, lábil, quase independente do texto que é suposto anunciar e ao qual parece fazer concorrência. Este artigo examina o caso emblemático de um título, "O quarto lado do triângulo", que parece duplamente enigmático : ao mesmo tempo Esfinge pelo teor críptico e Fénix pelo seu emprego iterativo num período de mais de uma dezena de anos. Encontramo-lo com efeito na nebulosa dos cadernos manuscritos, não datados e largamente inéditos, do escritor congolês, nomeando pelo menos quatro textos diferentes, de dimensão, período e género muito heterogéneos: uma novela, um romance 
inacabado, uma recolha de poemas, um esboço de ensaio autobiográfico. 0 dossier genético deste hipertítulo orienta-nos para a pista heurística de uma escrita hipertextual, para esse ponto cego onde se elabora, à força de partidas em falso, o processo re(tro)volucionário da contínua passagem do obsceno masculino ao secreto feminino que remata com o título final: Le Commencement des douleurs (O iniciar das dores).

\section{INDEX}

Mots-clés : dossier génétique, Labou Tansi Sony, paratexte, titre, fac-similé, manuscrit, tapuscrit, Le Quatrième côté du triangle, francophonie, études francophones

\section{AUTEUR}

\section{NICOLAS MARTIN-GRANEL}

NICOLAS MARTIN-GRANEL est agrégé de lettres classiques et diplômé de sciences politiques. Enseignant chercheur dans diverses universités africaines à Bamako, Nouakchott, Brazzaville, Yaoundé et Antananarivo, il a publié des ouvrages anthologiques (Rires noirs, Guide de littérature mauritanienne) et nombre d'articles critiques sur la littérature africaine, notamment congolaise. Ses recherches actuelles en génétique textuelle portent sur l'œuvre et les manuscrits inédits de Sony Labou Tansi dont il est l'éditeur scientifique (L'Autre Monde, L'Atelier de Sony Labou Tansi, Revue Noire, 1997 et 2005). Membre du comité de rédaction des revues Cahiers d'Études africaines et Riveneuve Continents, il est rédacteur en chef des Études littéraires africaines et chercheur associé à l'ITEM (CNRS-ENS) dans l'équipe Congo/Manuscrits francophones.

yanikos[arobase]aol.com 